\title{
Giant Intraperitoneal Liposarcoma: One Case Report
}

Samir Lafrouji ${ }^{1}{ }^{*}$, Faysal Alazaoui ${ }^{1}$, Hadj Omar El Malki ${ }^{1}$, Mountassir Chefchaouni ${ }^{1}$, Lahsen Ifrine ${ }^{1}$, Abdelkader Belkouchi ${ }^{1}$

\author{
${ }^{1}$ Surgery Department “A”, Ibn Sina Hospital, Mohammed-V University of Rabat, Morocco \\ DOI: $\underline{10.36347 / \text { sasjs.2021.v07i02.018 }}$ \\ | Received: 09.02.2021 | Accepted: 22.02.2021 | Published: 25.02.2021 \\ *Corresponding author: Samir Lafrouji
}

Abstract

Case Report

Liposarcoma is a primary malignancy and rare tumor developed most often from the soft tissue of the extremities. The intraperitoneal localization of this tumor is rare. It is usually unique, locally invasive, of late diagnosis and can reach a significant size and weight, producing a locally advanced tumor, called by some authors "giant intraperitoneal liposarcoma". CT scans and abdominal magnetic resonance imaging provide an accurate diagnosis. Surgical resection as large as possible is the only therapeutic mean. We report a rare case of a giant intraperitoneal liposarcoma, measuring $55 \mathrm{~cm}$ long, invading the left colon, the left part of the transverse colon, the left kidney and the left adrenal gland. Pathological, therapeutic and prognostic aspects of abdominal liposarcoma will be reviewed in this article.

Keywords: Liposarcoma - mesenchymal tumors - intra-peritoneal sarcoma.

Copyright $(\mathcal{C} 2021$ The Author(s): This is an open-access article distributed under the terms of the Creative Commons Attribution 4.0 International License (CC BY-NC 4.0) which permits unrestricted use, distribution, and reproduction in any medium for non-commercial use provided the original author and source are credited.

\section{INTRODUCTION}

Peritoneal liposarcoma is a malignant tumor with long asymptomatic development and late diagnosis, whose survival median does not exceed two years despite surgical excision [1]. Its frequency is estimated at $0.025 \%$ of digestive tumors operated on [2]. Its invasive potential is often underestimated. Macroscopically, it is a voluminous mass well limited and regular, soft and yellowish.

\section{CASE REPORT}

This is a 45-year-old patient, with no medical history, who initially consulted in urology for pain in the left lumbar fossa, increased abdominal girth and weight loss. The Physical examination noted a mucocutaneous pallor, edema of the lower limbs, an important abdominal masse, located at the level of the iliac left fossa and the left flank. The biology revealed an inflammatory syndrome, a microcytic hypochromic anemia with hemoglobin at 8.7 gr / dl, a hypoprotidemia at $57 \mathrm{gr} / 1$ and a hypoalbuminemia at 29. $\mathrm{gr} / 1$.

Abdominal ultrasound shows the presence of a large hypoechoic lesion with posterior, limited, intraperitoneal strengthening and extensive infiltration of mesenteric fat. Abdominal computed tomography (CT) revealed a large intraperitoneally mass of fatty density, heterogeneous in its contrast enhancement, surrounded by fine serpiginous vessels, in particular of the left renal compartment pushing back the left kidney, the digestive structures and the vascular axes (Picture 1 \& 2).

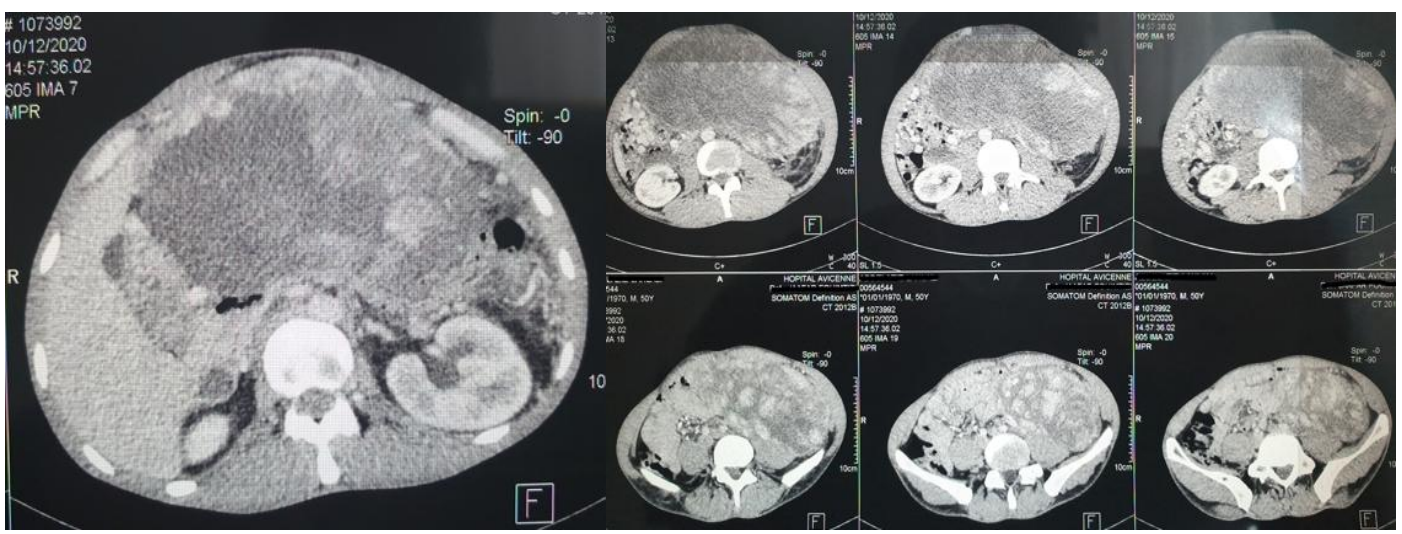

Picture-1 


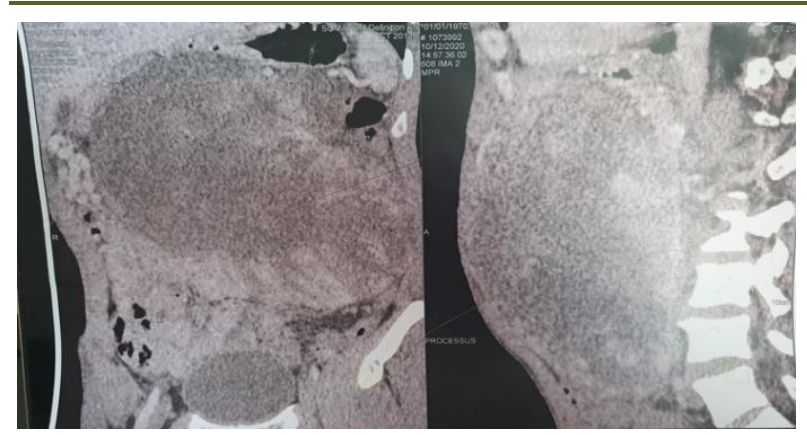

Picture-2

The patient underwent surgery. An enormous mass of the left renal lodge measuring $55 \mathrm{~cm}$, invading the left colon, the left part of the transverse colon, the left kidney and the left adrenal gland, reaching the spleen and overflowing on the large vessels on the right, was removed in monobloc (Picture-3).

Anatomopathological results showed that it is a dedifferentiated liposarcoma infiltrating the perirenal fat and the colonic wall, classified grade 3 by FNCLCC.
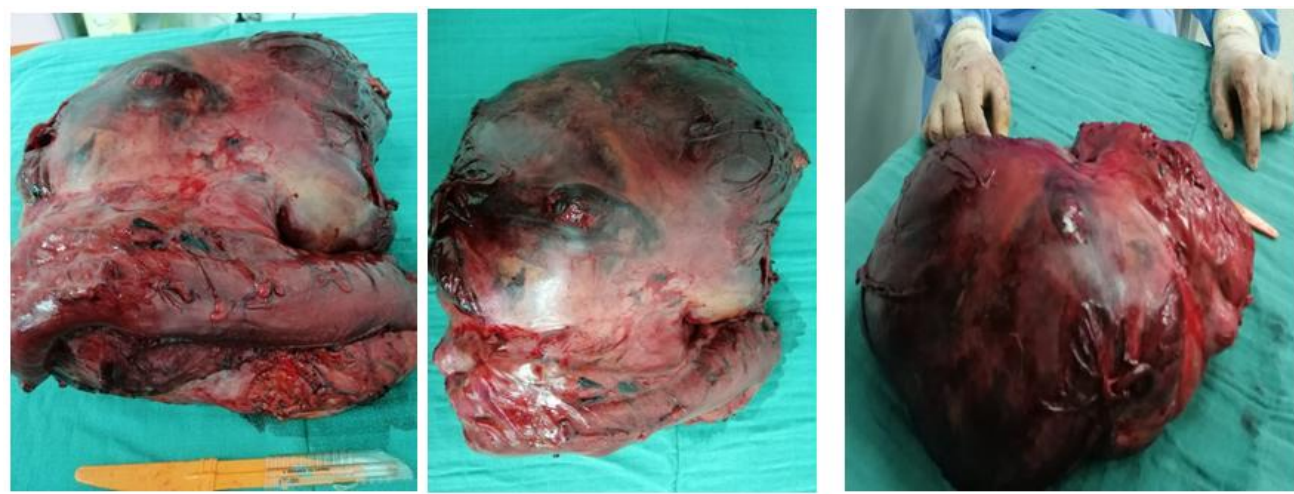

Picture-3: One-piece resection part of intra-peritoneal liposarcoma

\section{DISCUSSION}

Sarcomas are primary and rare mesenchymal tumors representing less than $1 \%$ of all malignant tumors [3]. Ten to $15 \%$ are localized at the level of the peritoneum and are dominated by liposarcoma, which represents around 40 to $50 \%$ of peritoneal sarcomas [3, 4]. It affects both sexes equally. The mean age at diagnosis is around the fifth decade, but the disease can affect all age groups [3, 4].

The slow increase in tumor volume and the complacency of the retroperitoneal space explain the long asymptomatic character of the tumor. The symptoms revealing the disease are not specific. They are due to the increase in tumor mass and the invasion or compression of nearby organs. They are dominated by abdominal pain or heaviness (50 to $80 \%$ ) and by the perception of an abdominal and / or pelvic mass (70 to $80 \%$ ). More rarely, these signs are associated with urinary or digestive disorders, deterioration in general condition and fever secondary to tumor necrosis $[3,5]$. The slow evolution of the tumor and the lack of specificity of the symptoms explain the diagnostic delay of the disease as well as the large size (which can reach $70 \mathrm{~cm}$ ) and the high weight (which can reach $40 \mathrm{~kg}$ ) carried by the tumor at the time of its discovery [3-5].

Liposarcoma is usually unique. Multiple synchronous locations are exceptional and reported as clinical facts in the literature. In our patient, there were one large tumor a long axis of $55 \mathrm{~cm}$.

Abdominal CT and magnetic resonance imaging (MRI) suggest the lipomatous nature of the lesion with great specificity. If liposarcoma poorly differentiated is easily diagnosed, especially by MRI, the distinction between lipoma and well-differentiated liposarcoma is a frequent dilemma because of the radiological similarities between these two lesions. However, certain radiological characteristics make it possible to differentiate them. Apart from the large size of the lesion, the presence of thick septa, nodular and / or globular lesions, non-fatty areas as well as the decrease in the adipose composition of the lesion strongly points to liposarcoma [6]. In addition to their role in diagnosis, CT and MRI make it possible to assess the locoregional extension of the tumor, its relationship with neighboring structures, the search for essentially hepatic metastases and the practice of guided biopsies, particularly in inoperable patients. The abdominal CT performed on our patient revealed a significant hypertrophy of the retroperitoneal fat, in particular of the left renal compartment, a large intra peritoneal mass pushing back the left kidney, the digestive structures and the vascular axes as well. These aspects evoked the diagnosis of liposarcoma.

The definitive diagnosis is made by the pathological examination of the tumor resection piece, which also allows the tumor to be classified according to its histological variety. Several histological varieties of increasing malignancy have been described. Welldifferentiated liposarcoma, myxoid liposarcoma, pleomorphic liposarcoma, round cell liposarcoma, and dedifferentiated liposarcoma. Well-differentiated liposarcoma with a better prognosis represents 30 to $35 \%$ of all liposarcomas. The myxoid form, which is the most frequent anatomopathological form $(50 \%)$ is 
clinically more malignant. Pleomorphic liposarcoma, round cell liposarcoma as well as dedifferentiated liposarcoma have a poor prognosis [7]. An association of two or three histological types within the same tumor is rare (5 to $10 \%$ ) leading to mixed-type liposarcoma [6, 8]. In our patient, histological examination concluded in a mixed type liposarcoma (dedifferentiated and myxoid).

Dedifferentiation of well-differentiated liposarcoma is a known complication, which increases with the duration of the tumor. The large dimensions of the liposarcomas do not in themselves represent a contraindication to an aggressive surgical approach. In fact, large surgical excision of the tumor, removing neighboring organs if necessary, is the only therapeutic alternative for these lesions [9, 10]. The difficulty of a curative tumor resection is due to its proximity, to its attachment and often to the invasion of vital structures. The value of adjuvant treatments is questionable. Radiotherapy used pre- or postoperatively appears to reduce the risk of recurrence. Due to its low chemosensitivity, the benefit of chemotherapy is limited. monobloc resection removing all of the tumor formation, the left kidney, the left adrenal gland, the left colon and the left part of the transverse colon was performed in our patient.

The main prognostic factor is the histological grade of the tumor. It is associated with local recurrence, recurrence of metastasis and overall survival [4]. Other prognostic factors have been implicated such as complete resection [11], the existence of synchronous metastases as well as neurovascular and bone involvement [4] overall survival is estimated at around $50 \%$ at five years and around $39 \%$ at ten years [4].

\section{REFERENCES}

1. Tsutsumi H, Ohwada S, Takeyoshi I, Izumi M, Ogawa T, Fukusato T, Morishita Y. Primary omental liposarcoma presenting with torsion: a case report. Hepato-gastroenterology. 1999 May $1 ; 46(27): 2110-2$.
2. Kurosaki Y, Tanaka YO, Itai Y. Welldifferentiated liposarcoma of the retroperitoneum with a fat-fluid level: US, CT, and MR appearance. Eur Radiol. 1998;8:474-5.

3. Antinori A, Antonacci V, Magistrelli P. Giant retroperitoneal liposar- coma. Am J Surg. 2002;184(1):56-7.

4. Stoeckle E, Coindre JM, Bonvalot S, Kantor G, Terrier P, Bonichon F, Nguyen Bui B. Prognostic factors in retroperitoneal sarcoma: a multivariate analysis of a series of 165 patients of the French Cancer Center Federation Sarcoma Group. Cancer: Interdisciplinary International Journal of the American Cancer Society. 2001 Jul 15;92(2):359-68.

5. Moualli SB, Mnif A, Amna MB, Hassine LB, Chebil M, Zermani R, Ayed M. Le liposarcome rétropéritonéal géant: à propos d'un cas. InAnnales d'urologie 2002 Dec 1 (Vol. 36, No. 6, pp. 372-375). Elsevier Masson.

6. Kransdorf MJ, Bancroft LW, Peterson JJ, Murphey MD, Foster WC, Temple HT. Imaging of fatty tumors: Distinction of lipoma and welldifferentiated liposarcoma. Radiology. 2002;224:99-104.

7. Bennani S, Debbagh A, Louahlia S, El Mrini M, Ben Jalloun S. Le liposarcome rétropéritonéal. À propos de deux cas. Ann Urol (Paris). 1995;29:154-8.

8. Tuoheti Y, Okada K, Miyakoshi N, Nishida J, Itoi E. Unusual variant of liposarcoma with multiple punctate calcifications. Skeletal Radiol. 2002;31(11):666-70.

9. Tsang A, Nash JR, Fordham MV, Hartley MN, Poston GJ. The man- agement of retroperitoneal liposarcoma with synchronous intraduode- nal sarcoma. Eur J Surg Oncol. 2003;29(6):515-8.

10. Bradley JC, Caplan R. Giant retroperitoneal sarcoma: a case report and review of the management of retroperitoneal liposarcoma. Am Surg. 2002;68(1):52-6.

11. Ferrario T, Karakousis CP. Retroperitoneal sarcomas. Grade and survival. Arch Surg. $2003 ; 138: 248-51$. 